\title{
PRL-3 improves colorectal cancer cell proliferation and invasion through IL-8 mediated glycolysis metabolism
}

\author{
HEYANG XU $^{1 *}$, YUJIE ZENG ${ }^{1 *}$, LU LIU ${ }^{1}$, QIAN GAO ${ }^{2}$, SHAOWEN JIN ${ }^{1}$, QIUSHENG LAN ${ }^{1}$, \\ WEI LAI ${ }^{1}$, XINGXI LUO $^{1}$, HENG WU $^{1}$, YONGLIANG HUANG ${ }^{1}$ and ZHONGHUA CHU ${ }^{1}$ \\ ${ }^{1}$ Department of Gastrointestinal Surgery, Sun Yat-sen Memorial Hospital, Sun Yat-sen University, \\ Guangzhou, Guangdong 510120; ${ }^{2}$ Department of Obstetrics, The Third Affiliated Hospital \\ of Sun Yat-sen University, Guangzhou, Guangdong 510120, P.R. China
}

Received May 23, 2017; Accepted July 19, 2017

DOI: 10.3892/ijo.2017.4090

\begin{abstract}
Phosphatase of regenerating liver-3 (PRL-3) has been found to be overexpressed in liver metastases of colorectal cancer and rarely expressed in primary tumors, which plays an important role in the metastasis of colorectal cancer cells. Metabolism reprogramming has been found to be a hallmark of cancer cells, and aerobic glycolysis is a metabolic adaption for cancer cells and promotes cell proliferation. However, the association between PRL-3 and glycolysis in colorectal cancer cells is not well understood. In the present study, we explored the association between PRL-3 and glycolysis. We found that PRL-3 improved colorectal cancer cell glucose assumption, lactate production and reduced intracellular ROS levels. Besides, PRL-3 improved the expression of Glut1, HK2, PKM2 and LDHA, which are important glycolysis related molecules and enzymes. Moreover, we explored IL-8 mediated enhancement of glycolysis by PRL-3. More importantly, the proliferation and invasion of colorectal cancer cells were enhanced significantly by PRL-3 through improving glycolysis. Taken together, these results implicated the important role of PRL-3 in glycolysis metabolism through improving IL-8 secretion in colorectal cancer cells, and PRL-3 mediated glycolysis contributed to the promotion of cancer metastasis.
\end{abstract}

\section{Introduction}

Colorectal cancer is one of the most common cancers in the world. Patients in advanced stages with metastatic lesions

Correspondence to: Professor Zhonghua Chu and Dr Yongliang Huang, Department of Gastrointestinal Surgery, Sun Yat-sen Memorial Hospital, Sun Yat-sen University, Guangzhou, Guangdong 510120, P.R. China

E-mail: chu9009@163.com

E-mail: 329659366@qq.com

*Contributed equally

Key words: phosphatase of regenerating liver-3, colorectal cancer, IL-8, glycolysis metabolism appear to have a poor prognosis (1). Clinical studies have found that colorectal cancer cells prefer to metastasize to the liver over other organs, which results in poor prognosis. Therefore, it is important to identify and understand the factors involved in the progression of colorectal cancer metastasis to the liver.

PRL-3 belongs to the family of protein tyrosine phosphatases (PTPs), which has been demonstrated to play an important role in colorectal cancer metastasis in the liver (2). PTPs regulate phosphorylation of many important signaling molecules that are involved in cell proliferation, migration and prognosis (3). PRL-3 is normally expressed in heart and skeletal muscle. However, studies have found that PRL-3 is significantly overexpressed in metastatic cells and is moderately expressed in primary lesions of colorectal cancer (4). Moreover, the expression of PRL-3 in primary colorectal cancer lesions indicates poor prognosis and shortened survival (5). Therefore, PRL-3 appears to be a biomarker for colorectal cancer, and, in particular, a biomarker for colorectal cancer liver metastasis. However, the mechanisms of regulating liver metastasis are still uncertain. Our previous research found that PRL-3 promoted colorectal cancer cell proliferation through TNF- $\alpha$ secretion, which also induced the activation of a $\mathrm{Ca}^{2+}$-activated $\mathrm{K}^{+}$channel (KCNN4) (6). We demonstrated that PRL-3 facilitated epithelial mesenchymal transition (EMT) in colorectal cancer cells (7), indicating that PRL-3 strongly influenced the biological characteristics of tumor cells. Moreover, we studied the tumor microenvironment and found that PRL-3 integrated with tumor associated macrophages (TAMs), induced TAM secreting inflammatory cytokines such as IL-6 and IL-8, which then enhanced colorectal cancer cell invasion (8).

Tumor cell growth and proliferation require large quantities of bioenergy and biomaterials. In recent years, increasing number of studies have found that an important hallmark of cancer cells is metabolism reprogramming, which was first proposed by Warburg (termed the Warburg effect) in the 1920s (9,10). Unlike normal cells, most cancer cells exhibit a high rate of glycolysis rather than oxidative phosphorylation and therefore produce large amounts of lactate, leading to a decrease in extracellular $\mathrm{pH}$, which facilitates cancer cell removal (11). The activation of glycolytic pathways promotes tumor cells to adapt to fast proliferation. Furthermore, glycolysis-associated enzymes and molecules are highly 
Table I. Oligonucleotide sequence of qRT-PCR primers.

\begin{tabular}{llll}
\hline Gene & \multicolumn{1}{c}{ Forward primer } & \multicolumn{1}{c}{ Reverse primer } & Amplicon \\
\hline GAPDH & AATGGGCAGCCGTTAGGAAA & GCGCCCAATACGACCAAATC & 168 \\
PRL-3 & ACACATGCGCTTCCTCATCA & GTCACTTCACACACACGCAC & 111 \\
Glut1 & GGCTTCTCCAACTGGACCTC & CCGGAAGCGATCTCATCGAA & 176 \\
HK2 & CAAGAAGCTCCCACTGGGTT & CAACGTCTCTGCCTTCCACT & 122 \\
PKM2 & GTCTGGGAGGAAAGTCGCTC & GGCGGAAGGACACAGATTCA & 104 \\
LDHA & CATGGCCTGTGCCATCAGTA & AGATATCCACTTTGCCAGAGACA & 158 \\
IL-8 & CCACCGGAAGGAACCATCTC & TTCCTTGGGGTCCAGACAGA & 279
\end{tabular}

expressed in tumor cells, which also play an important role in cancer (12).

Inflammation is an important risk factor for colorectal cancer, but the mechanisms underlying this effect of inflammation on colorectal cancer cells are still not fully understood. It has been indicated that tumor associated inflammation may affect the proliferation, metastasis and angiogenesis of tumor cells (13). Notably, our previous study also found that inflammatory cytokines IL-6 and IL-8, which were secreted by TAMs, enhanced colorectal cancer cells invasion (8). However, the association between inflammatory cytokines and glycolysis metabolism is still uncertain.

In the present study, we aimed to determine whether PRL-3 is involved in the metabolism reprogramming of colorectal cancer cells. This investigation revealed that PRL-3 promotes glycolysis through secretion of IL-8 in colorectal cancer cells, leading to an increase of glucose consumption and lactate production, reduced intercellular reactive oxygen species (ROS) levels and induced overexpression of glycolysis enzymes and molecules, contributing to enhanced tumor cell proliferation and invasion.

\section{Materials and methods}

Samples and patients. Colorectal cancer cell samples were collected from 47 patients admitted to the Department of Gastroenteropancreatic Surgery of Sun Yat-sen Memorial Hospital, Sun Yat-sen University, between 2013 and 2016. Specimens were collected immediately after tumor removal. All samples were collected with informed consent according to the Internal Review and the Ethics Boards of the Sun Yat-sen Memorial Hospital of Sun Yat-sen University. The protocol was approved by the Ethics Committee of Sun Yat-sen Memorial Hospital.

Cell cultures and treatments. LoVo colorectal cancer cells were purchased from the Shanghai Cell Bank of the Chinese Academy of Sciences (Shanghai, China). Cells were transfected with PAcGFP-PRL-3 (LoVo-P) or PAcGFP (LoVo-C) using Lipofectamine 3000. Cells were stored at Sun Yat-sen Memorial Hospital (6). Cells were cultured in RPMI-1640 medium and 10\% fetal bovine serum (FBS), with $100 \mathrm{mg} / \mathrm{ml}$ penicillin. The cells were incubated at $37^{\circ} \mathrm{C}, 5 \% \mathrm{CO}_{2}$ in a humidified atmosphere.

Reagents and antibodies. Lipofectamine 3000 was purchased from Sigma-Aldrich (St. Louis, MO, USA). Fetal bovine serum
(FBS) was purchased from Biological Industries (Kibbutz Beit Haemek, Israel). RPMI was purchased from Invitrogen (Carlsbad, CA, USA). TRIzol and Prime Script RT were purchased from Takara Bio (Dalian, China). The siRNA was purchased from Shanghai GenePharma, Co., Ltd. (Shanghai, China). Antibodies against GAPDH (cat. no. ab8245), IL-8 (cat. no. ab18672), Glut1 (cat.no. ab115730), PKM2 (cat.no. ab38237), HK2 (cat. no. ab104836), LDHA (cat. no. ab125683) were purchased from Abcam (Cambridge, MA, USA).

Western blot assay. Cells were washed with phosphatebuffered saline (PBS) and then lysed on ice with RIPA buffer containing 1\% PMSF. The Bradford assay was used to detect protein concentration. Denatured proteins were separated by $10 \%$ sodium dodecyl sulfate-polyacrylamide gel electrophoresis, transferred to PVDF membranes and then blocked in $5 \%$ non-fat milk. Membranes were washed 3 times with Trisbuffered saline $+0.1 \%$ Tween-20 (TBST), incubated overnight at $4^{\circ} \mathrm{C}$ with relevant primary antibodies, and then washed and incubated with horseradish peroxidase-conjugated secondary antibodies for $1 \mathrm{~h}$ at room temperature. Labeled proteins and relative band intensities were visualized and measured with Quantity One software (Bio-Rad Laboratories, Hrercules, CA, USA).

RNA extraction and real-time quantitative $R T-P C R$. Total RNA was isolated using TRIzol reagent, and 500 ng RNA was reverse transcribed using PrimeScript RT according to the manufacturer's protocol. Quantitative real-time RT-PCR was performed using the LightCycler 480 (Roche, Basel, Switzerland) and SYBR assays (Takara Bio). Primers were designed to detect GAPDH, PRL-3, Glut1, PKM2, HK2, LDHA and IL-8. The primers used for qRT-PCR are shown in Table I. Each sample contained 1X SYBR Premix Ex Taq ${ }^{\mathrm{TM}}, 0.2 \mu \mathrm{M}$ of each forward and reverse primer and 500 ng template cDNA in a final volume of $20 \mu \mathrm{l}$. Cycling parameters were set as follows: denaturation at $95^{\circ} \mathrm{C}$ for $30 \mathrm{sec}$, followed by 40 amplification cycles $\left(95^{\circ} \mathrm{C}\right.$ for $5 \mathrm{sec}$ and $60^{\circ} \mathrm{C}$ for $\left.20 \mathrm{sec}\right)$. For relative quantification, $2^{-\Delta \mathrm{Ct}}$ was used to calculate the fold change in gene expression. All experiments were performed in triplicate.

Glycolysis consumption and lactate production. Glucose and lactate assay kits were purchased from Sigma-Aldrich to determine the concentrations of glucose and lactate in the culture medium, respectively. Cells were seeded on 6-well plates at a density of $1 \times 10^{5}$ cells/well and the medium was 
changed to DMEM after incubation overnight. The concentrations of glucose and lactate were measured according to the manufacturer's protocol.

Measurement of intracellular reactive oxygen species (ROS). Intracellular ROS levels were detected by $\mathrm{H}_{2}$ DCF-DA (Invitrogen). The cells were cultured in a 96-well plate. Cells were washed with PBS before incubation with $\mathrm{H}_{2}$ DCF-DA for $30 \mathrm{~min}$, and ROS levels were examined at excitation and emission wavelength of 485 and $520 \mathrm{~nm}$. Cell numbers were normalized before the measurement.

Inflammatory cytokine array analysis. The RayBio Cytokine Analysis array (RayBiotech, Norcross, GA, USA), consisting of 40 different inflammatory cytokine antibodies spotted onto a membrane, was used in the present study. Cytokine array membranes were blocked for $30 \mathrm{~min}$ and then incubated with samples at $37^{\circ} \mathrm{C}$ for $1 \mathrm{~h}$. Then membranes were washed and incubated with diluted biotin-conjugated antibodies at $37^{\circ} \mathrm{C}$ for $2 \mathrm{~h}$. After the membranes were washed, 1,000-fold diluted horseradish peroxidase-conjugated streptavidin was added and incubation was continued for $2 \mathrm{~h}$. Membranes were then washed thoroughly and exposed to detection buffer in the dark. By comparing the signal intensities, relative expression levels of cytokines were made. The intensities of signals were quantified by densitometry.

Immunohistochemistry. Using primary antibodies against PRL-3, Glut1, PKM2, HK2, LDHA and IL-8, the tissue slides were incubated overnight at room temperature. Secondary staining with Alexa Fluor 555 conjugated donkey anti-rabbit and Alexa Fluor 488 conjugated goat anti-mouse secondary antibodies was performed at room temperature for $60 \mathrm{~min}$. Images were taken with a Zeiss LSM 700 laser scanning microscope (Carl Zeiss, Oberkochen, Germany) with a core data acquisition system (Applied Precision, Bratislava, Slovakia). For control experiments, primary antibody was substituted with normal rabbit serum.

Wound scratch test and invasion assays. Cell migration was measured by the movement of cells into a scraped area created by the tip of a $200 \mu \mathrm{l}$ pipette. The degree of 'wound closure' was examined after 24 and $48 \mathrm{~h}$. After cell adherence, the remaining gap was then measured using light microscopy and quantified. Invasion assays were performed using $10^{5} \mathrm{cells} /$ well added to a Matrigel invasion chamber. FBS was added to the lower chamber, and the number of cells invaded from the top chamber after 24 and $48 \mathrm{~h}$ was measured with a spectrophotometer.

Statistical analysis. Statistical analysis was performed using the SPSS. Data from three separate experiments were reported as the means \pm SD. Statistical significance between the samples was assessed by the Student's-t test where $\mathrm{P}<0.05$ was considered to be statistically significant.

\section{Results}

PRL-3 promotes glycolysis in colorectal cancercells. Tumorcells reprogram their metabolism from oxidative phosphorylation to
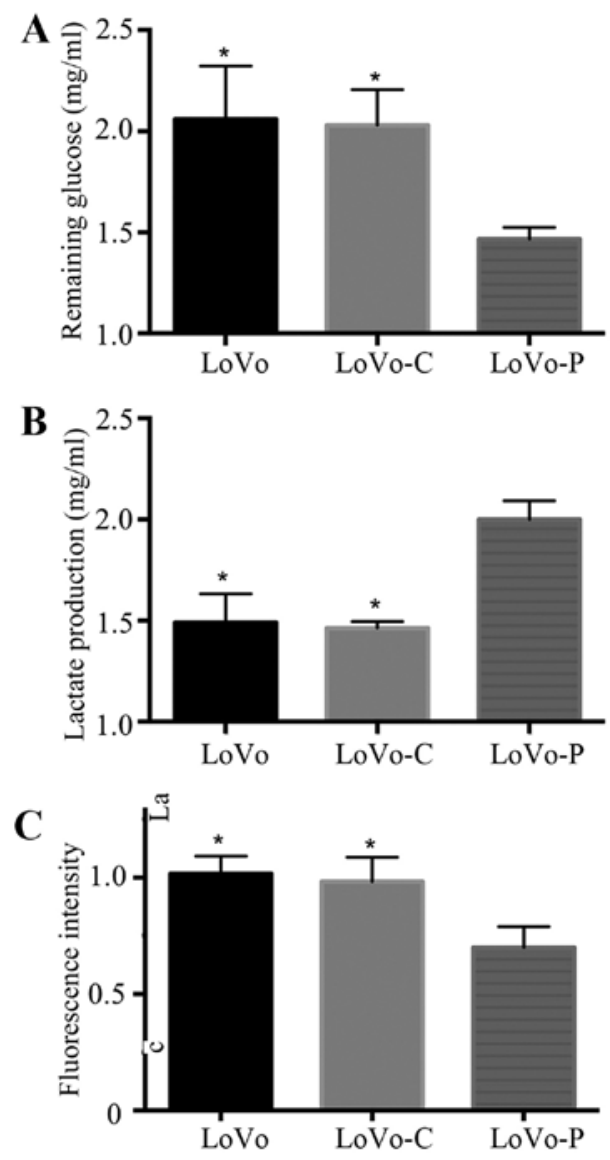

Figure 1. PRL-3 promotes glycolysis in colorectal cancer cells. (A) The remaining glucose in LoVo, LoVo-C and LoVo-P cell culture medium. (B) Lactate production in LoVo, LoVo-C and LoVo-P cell culture medium. (C) Intercellular ROS levels in LoVo, LoVo-C and LoVo-P cells, ${ }^{*} \mathrm{P}<0.05$.

glycolysis to meet huge energy demands and for large amounts of biomass (14). A glycolytic feature is often characterized by glucose assumption, lactate production and reduced intracellular ROS levels. To examine the role of PRL-3 in glycolysis of colorectal cancer cells, we used glucose consumption assays, lactate production assays and intracellular ROS measurement assays to analyze glycolysis in PRL-3 overexpressed colorectal cancer cells (LoVo-P), control cells (LoVo-C) and wild-type cells (LoVo). We found that overexpression of PRL-3 promoted glucose consumption, lactate production and reduced the intracellular ROS levels. However, LoVo-C and LoVo cells did not exhibit the same trends (Fig. 1).

PRL-3 promotes Glut1, HK2, PKM2 and LDHA expression in colorectal cancer cells. The progression of glycolysis contains more than ten metabolic reactions that are catalyzed by a number of enzymes or molecules. For example, Glutl transports glucose across the plasma membrane, which plays an important role in rate-limiting glucose metabolism. Additionally, HK2, PKM2 and LDHA are important rate-limiting enzymes in glycolysis. To examine whether glycolysis-associated molecules and enzymes may be regulated by PRL-3 in colorectal cancer cells, we analyzed mRNA expressions of Glut1, HK2, PKM2 and LDHA. Compared to LoVo-C and LoVo cells, the data showed that expression of Glut1, HK2, PKM2 and LDHA were all increased in LoVo-P cells (Fig. 2A). Consistent with 

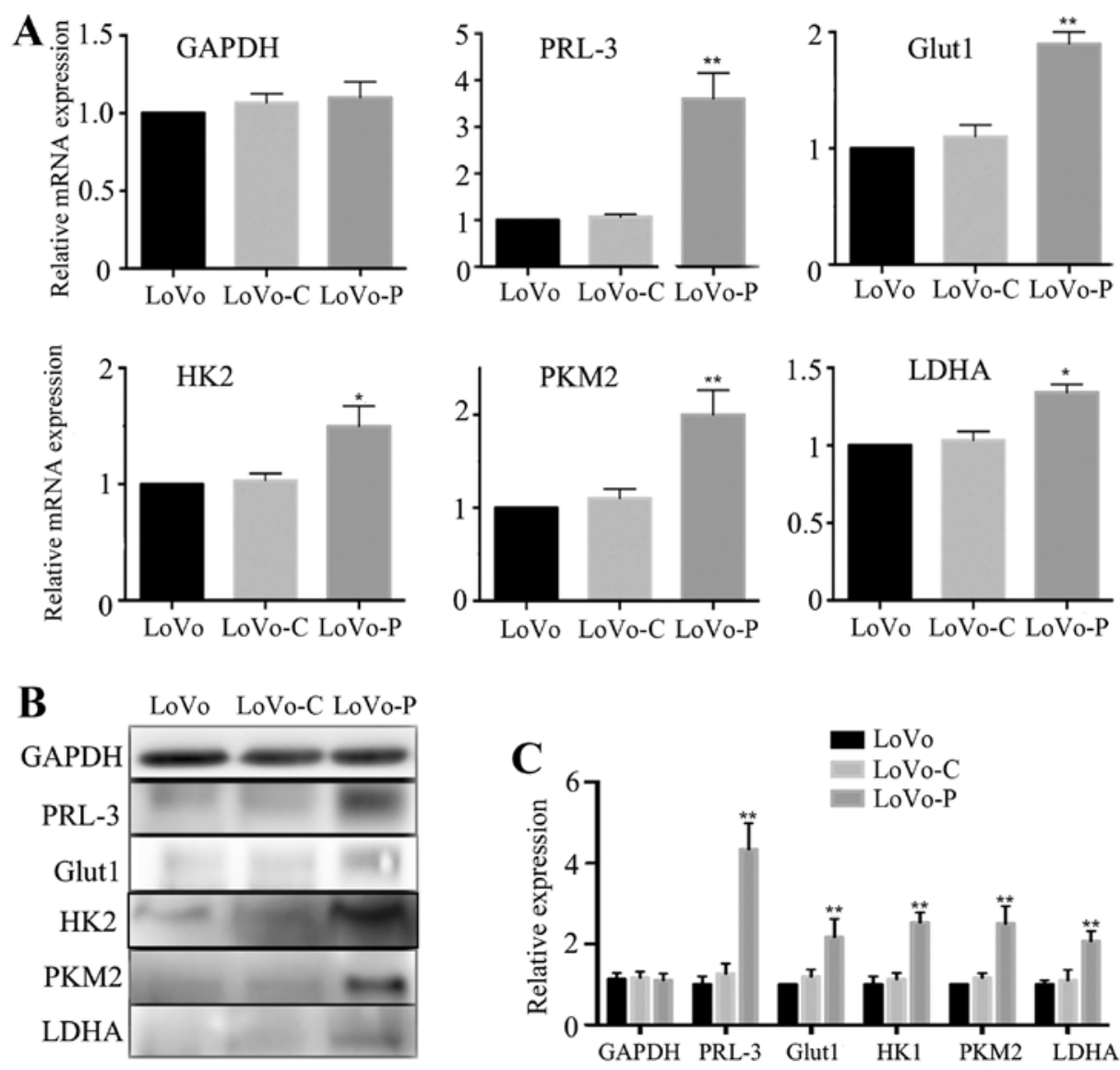

Figure 2. PRL-3 promotes Glut1, HK2, PKM2 and LDHA expression in colorectal cancer cells. (A) GAPDH, PRL-3, Glut1, HK2, PKM2 and LDHA mRNA expression levels in LoVo, LoVo-C and LoVo-P cells. (B and C) GAPDH, PRL-3, Glut1, HK2, PKM2 and LDHA protein expression levels in LoVo, LoVo-C and LoVo-P cells. ${ }^{*} \mathrm{P}<0.05,{ }^{* *} \mathrm{P}<0.01$.

\section{A}
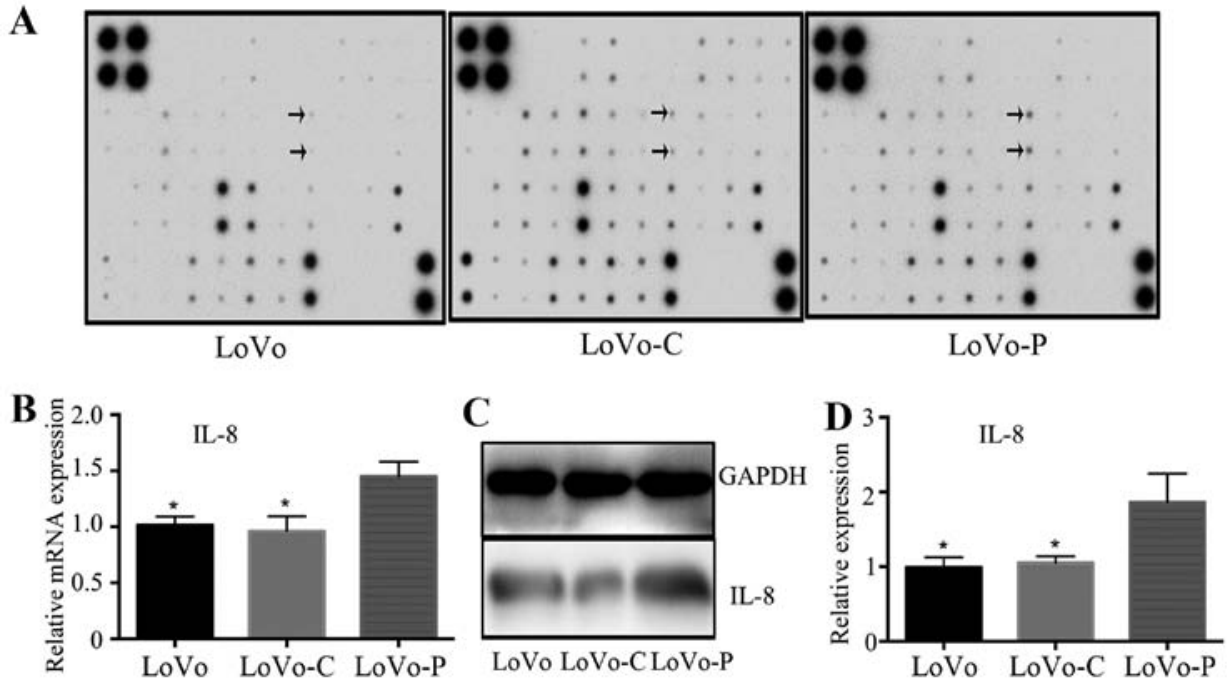

Figure 3. PRL-3 improves IL-8 expression in colorectal cancer cells. (A) Inflammatory cytokine profiles of LoVo, LoVo-C and LoVo-P cells. (B) IL-8 mRNA expression in LoVo, LoVo-C and LoVo-P cells. (C and D) IL-8 protein expression in LoVo, LoVo-C and LoVo-P cells. " $\mathrm{P}<0.05$.

these results, western blot analysis also showed the same trends (Fig. 2B and C).

PRL-3 improves IL-8 expression in colorectal cancer cells. We next explored the inflammatory cytokine expression in supernatants by using inflammatory cytokine antibody array to find the expression differences between LoVo-P, LoVo-C and LoVo cells. Each membrane contained 40 inflammatory cytokines (Table II), relative inflammatory cytokine expression was compared between between LoVo-P, LoVo-C and 
Table II. Inflammatory cytokines on the membrane.

\begin{tabular}{|c|c|c|c|c|c|c|c|c|c|c|c|c|}
\hline & A & B & $\mathrm{C}$ & D & E & F & $\mathrm{G}$ & $\mathrm{H}$ & I & $\mathrm{J}$ & K & $\mathrm{L}$ \\
\hline 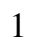 & POS & POS & NEG & NEG & Eotaxin & Eotaxin-2 & G-CSF & GM-CSF & ICAM1 & IFN- $\gamma$ & I-309 & IL-1 $\alpha$ \\
\hline 2 & POS & POS & NEG & NEG & Eotaxin & Eotaxin-2 & G-CSF & GM-CSF & ICAM1 & IFN- $\gamma$ & I-309 & IL-1 $\alpha$ \\
\hline 3 & IL-1 $\beta$ & IL-2 & IL-3 & IL-4 & IL-6 & IL-6sR & IL-7 & IL-8 & IL-10 & IL-11 & IL-12p40 & IL-12p70 \\
\hline 4 & $\mathrm{IL}-1 \beta$ & IL-2 & IL-3 & IL-4 & IL-6 & IL-6sR & IL-7 & IL-8 & IL-10 & IL-11 & IL-12p40 & IL-12p70 \\
\hline 5 & IL-13 & IL-15 & IL-16 & IL-17 & IP-10 & MCP-1 & MCP-2 & M-CSF & MIG & MIP- $1 \alpha$ & MIP- $1 \beta$ & MIP-1ठ \\
\hline 6 & IL-13 & IL-15 & IL-16 & IL-17 & IP-10 & MCP-1 & MCP-2 & M-CSF & MIG & MIP- $1 \alpha$ & MIP- $1 \beta$ & 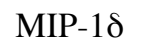 \\
\hline 7 & RANTES & TGF- $\beta$ & TNF- $\alpha$ & TNF- $\beta$ & sTNF-RI & sTNF-RII & PDGF-BB & TIMP-2 & BLANK & BLANK & NEG & POS \\
\hline 8 & RANTES & TGF- $\beta$ & TNF- $\alpha$ & TNF- $\beta$ & sTNF-RI & sTNF-RII & PDGF-BB & TIMP-2 & BLANK & BLANK & NEG & POS \\
\hline
\end{tabular}
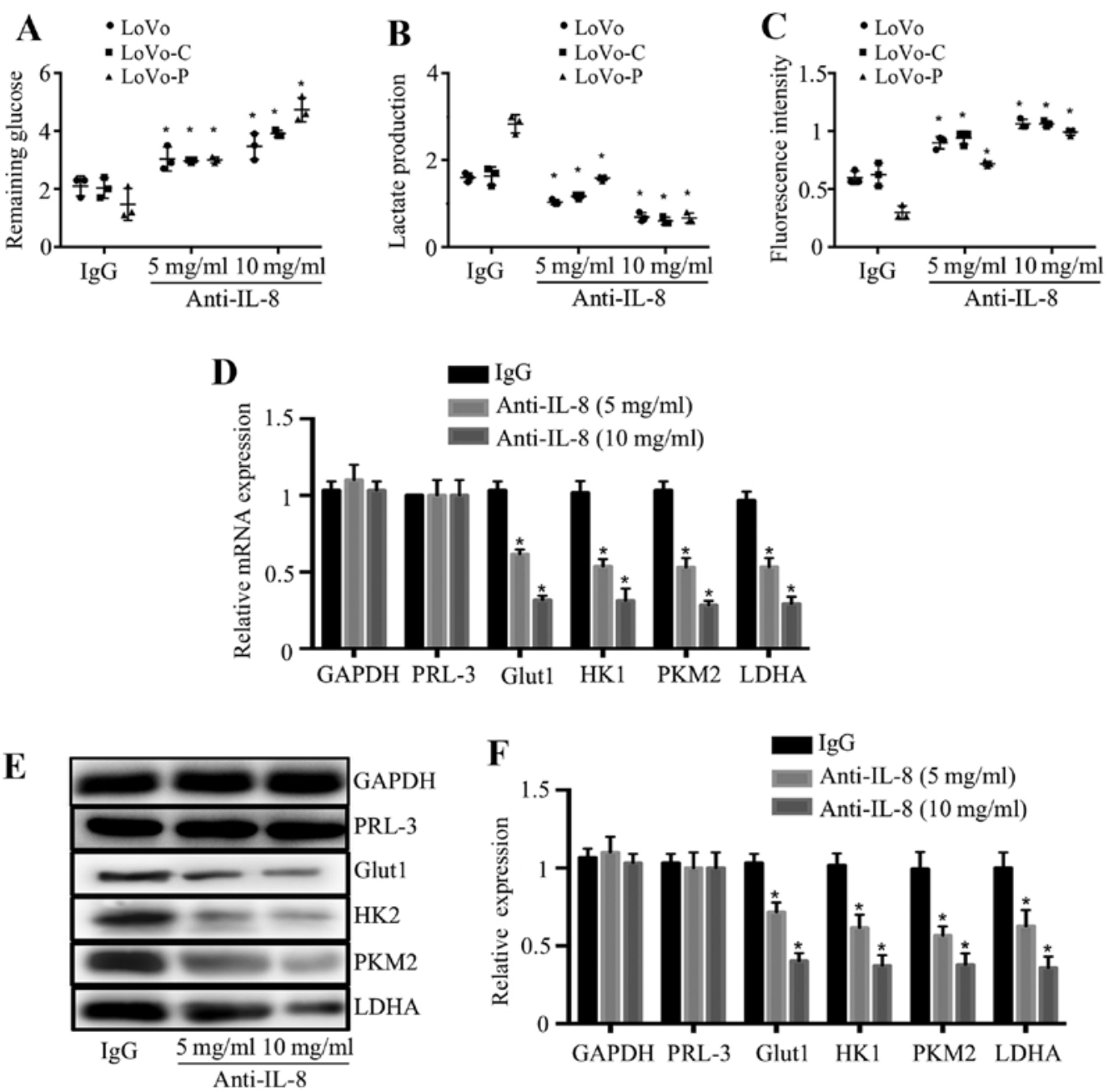

Figure 4. IL-8 mediates the promotion of glycolysis in colorectal cancer cells. (A-C). Remaining glucose, lactate production and intracellular ROS levels were detected in LoVo, LoVo-C, LoVo-P cells which were pretreated with an isotype-matched IgG control (IgG, $10 \mathrm{mg} / \mathrm{ml}$ ) or anti-IL-8 antibody at 5 or $10 \mathrm{mg} / \mathrm{ml}$. (D) mRNA expression of GAPDH, PRL-3, Glut1, HK2, PKM2 and LDHA in LoVo-P cells which were pretreated with anti-IL-8 antibody at 5 or $10 \mathrm{mg} / \mathrm{ml}$. (E and F) Protein expression of GAPDH, PRL-3, Glut1, HK2, PKM2 and LDHA in LoVo-P cells which were pretreated with anti-IL-8 antibody at 5 or $10 \mathrm{mg} / \mathrm{ml}$. ${ }^{*} \mathrm{P}<0.05$.

LoVo cells separately. Results showed significant upregulation of IL-8 expression in LoVo-P cells (Fig. 3A). RT-PCR showed that IL-8 gene was upregulated in LoVo-P cells (Fig. 3B). In line with the mRNA level, the protein level of IL- 8 showed the same trends (Fig. 3C and D). These data suggested that PRL-3 improved the expression of IL- 8 in colorectal cancer cells.
IL- 8 mediates the promotion of glycolysis in colorectal cancer cells. To explore whether IL- 8 mediates the promotion of glycolysis, we used anti-IL- 8 antibody to neutralize IL- 8 function. The addition of anti-IL- 8 antibody to the culture medium increased the remaining glucose, reduced the lactate production and increased intracellular ROS 

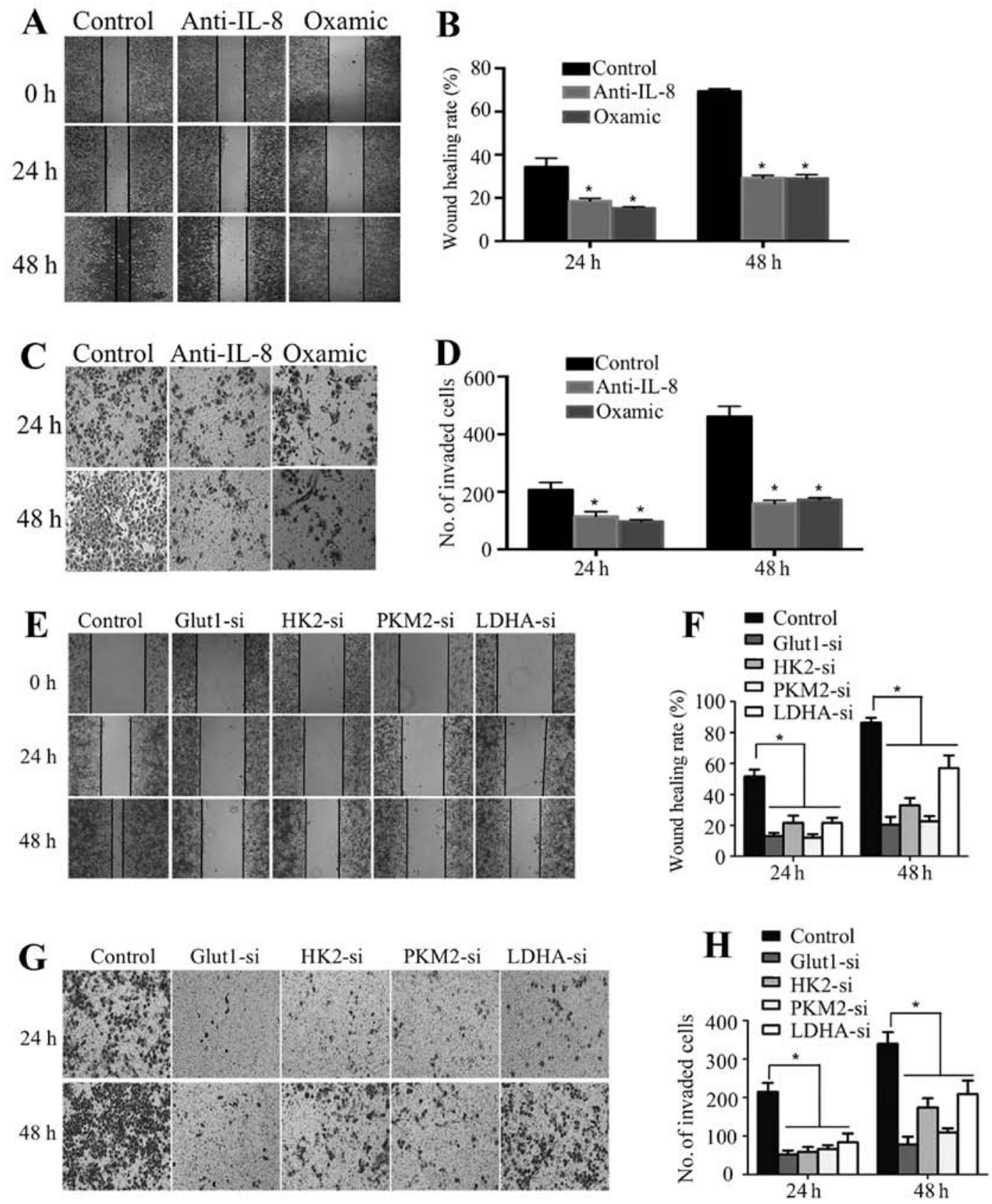

Figure 5. PRL-3 improves the growth and invasion in colorectal cancer cells through glycolysis. (A-D) Treatment of PBS (Control) or anti-IL-8 (10 mg/ml) or oxamic $90 \mathrm{mM}$ acid (Oxamic) in LoVo-P cells for 2 days, then migration and invasion were tested through wound healing assays and Matrigel invasion assays. (E-H) PBS, Glut1-siRNA, HK2-siRNA, PKM2-siRNA or LDHA-siRNA was pretreated in LoVo-P cells, respectively. Then, migration and invasion were tested through wound healing assays and Matrigel invasion assays. " $\mathrm{P}<0.05$.

levels of cancer cells in a dose-dependent manner, whereas an isotype-matched $\operatorname{IgG}(10 \mu \mathrm{g} / \mathrm{ml})$ did not have same effects (Fig. 4A-C), indicating the role of IL-8 in colorectal cancer cells glycolysis. In order to examine whether PRL-3 improves glycolysis related molecules and enzymes through IL-8, we examined the effect of IL-8 on the expression of Glut1, HK2, PKM2 and LDHA in LoVo-P cells. RT-PCR and western blot analysis showed these molecules and enzymes were significantly reduced after anti-IL-8 antibody was added into the culture medium (Fig. 4D and F).

PRL-3 improves growth and invasion via glycolysis in colorectal cancer cells. Lactate and glycolysis-associated enzymes and molecules have been found to play important roles in improving cancer cell metastasis (15-19). To identify the role of PRL-3 induced glycolysis through IL-8 on colorectal cancer cell growth and invasion, we inhibited lactate by pretreating colorectal cancer cells with oxamic acid, or we inhibited Glut1, HK2, PKM2 or LDHA expression by siRNA, or we inhibited IL- 8 by pretreating anti-IL- 8 antibody. We found that LoVo-P cells exhibited decreased motility and invasion when oxamic acid or anti-IL-8 antibody was added (Fig. 5A-D). Moreover, our data also showed decreased motility and invasion of LoVo-P cells when the expression of Glut1, HK2, PKM2 or LDHA was inhibited (Fig. 5E-H). 

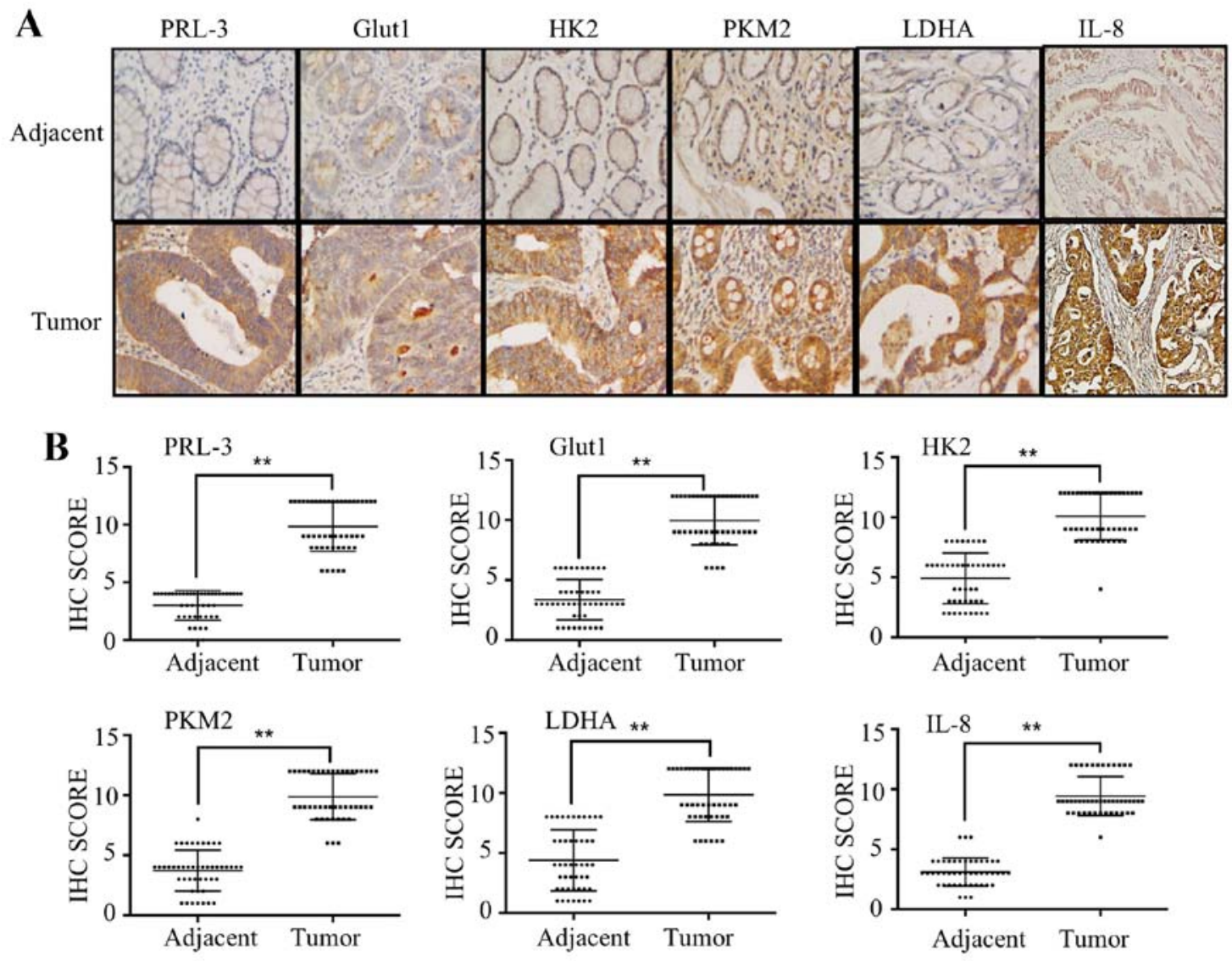

Figure 6. Correlation between PRL-3 and Glut1, HK2, PKM2, LDHA, IL-8 in CRC patients. (A) The expression of PRL-3, Glut1, HK2, PKM2, LDHA and IL-8 in human adjacent normal and tumor tissues was evaluated by immunohistochemistry. (B) IHC scores of PRL-3, Glut1, HK2, PKM2, LDHA and IL-8 in 47 tumor and corresponding adjacent normal tissues. ${ }^{*} \mathrm{P}<0.05$.

Correlation between PRL-3 and Glut1, HK2, PKM2, LDHA and IL- 8 in $C R C$ patients. To explore the association between PRL-3, IL-8, Glut1, HK2, PKM2 and LDHA in clinical patient tissues, we performed IHC and scored the results of 47 patients with colorectal cancer. We first analyzed the expression of PRL-3 in tissues from clinical colorectal carcinoma samples. Consistent with our previous research, PRL-3 was rarely expressed in adjacent normal colorectal lesions but was overexpressed in colorectal carcinomas lesions. Furthermore, we analyzed the protein expression of Glut1, HK2, PKM2, LDHA and IL-8 and found that they were overexpressed in tumor tissues and positively correlated with PRL-3 expression (Fig. 6A). IHC scores of PRL-3, Glut1, HK2, PKM2, LDHA and IL-8 were remarkably higher in tumor tissues than those in normal adjacent tissues (Fig. 6B and Table III).

\section{Discussion}

Reprogrammed metabolism, which fuels tumor cells replication, growth and invasion, was added to the hallmarks of cancer (20). Many studies have explored the mechanisms of tumor cell unlimited growth and altered metabolism. In the present study, we found that PRL-3 improves glycolysis of colorectal cancer cells, which contributes to cancer cells proliferation and invasion in vitro. Our previous research revealed that inflammatory cytokine IL-8, which was secreted by tumor associated macrophage, promoted colorectal cancer cell invasion (8). Our current experiments showed that PRL-3 improved IL-8 expression in colorectal cancer cells, and IL-8 participates in the promotion of glycolysis by PRL-3. To the best of our knowledge, this is the first report indicating the association between PRL-3 and tumor metabolism reprogram, furthermore, our research uncovered the role of inflammatory cytokine IL-8 in glycolysis.

Various research has been made into the key steps of metastatic process influenced by PRL-3. For example, PRL-3 repressed various target genes which participate in cell cycle arrest to give cell unlimited proliferative advantage (21). PRL-3 promotes PI3K-AKT activity, which is an important driver of cell proliferation and survival (22). PRL-3 has been associated in regulation of focal adhesion components, such as Src, integrin and paxillin, which induced cell motility (23). PRL-3 promoted cell invasion by increasing MMP2 activity (23), induced EMT by acting upstream of PI3K/AKT signaling (24). PRL-3 increased the expression of VEGF and promoted tumor cell angiogenesis (25). However, little attention has been given to the relationship between PRL-3 and tumor metabolism reprogram. In the present study, remaining glucose in the culture medium of LoVo-P cells is less than LoVo and LoVo-C cells, which means LoVo-P cells consumed more glucose. The culture medium did not contain FBS to eliminate possible interference caused by cell growth rate. Besides, lactate production level was 
Table III. Association of PRL-3 and Glut1, HK2, PKM2, LDHA, IL-8 expression in 47 colorectal cancer patients.

\begin{tabular}{|c|c|c|}
\hline & Adjacent tissues & Tumor tissues \\
\hline Number & 47 & 47 \\
\hline \multicolumn{3}{|l|}{ PRL-3 expression } \\
\hline No staining & 3 & 0 \\
\hline Weak staining & 44 & 0 \\
\hline Intermediate staining & 0 & 14 \\
\hline Strong staining & 0 & 43 \\
\hline IHC score, mean $\pm \mathrm{SE}$ & $3.0 \pm 1.3$ & $9.8 \pm 2.1$ \\
\hline P-value & $<0.05$ & \\
\hline \multicolumn{3}{|l|}{ Glut1 expression } \\
\hline No staining & 0 & 0 \\
\hline Weak staining & 37 & 4 \\
\hline Intermediate staining & 10 & 6 \\
\hline Strong staining & 0 & 37 \\
\hline $\mathrm{IHC}$ score, mean $\pm \mathrm{SE}$ & $3.4 \pm 1.7$ & $9.9 \pm 2.0$ \\
\hline P-value & $<0.05$ & \\
\hline \multicolumn{3}{|l|}{ HK2 expression } \\
\hline No staining & 0 & 0 \\
\hline Weak staining & 20 & 1 \\
\hline Intermediate staining & 27 & 10 \\
\hline Strong staining & 0 & 36 \\
\hline $\mathrm{IHC}$ score, mean $\pm \mathrm{SE}$ & $4.9 \pm 2.1$ & $10.1 \pm 2.0$ \\
\hline P-value & $<0.05$ & \\
\hline \multicolumn{3}{|l|}{ PKM2 expression } \\
\hline No staining & 0 & 0 \\
\hline Weak staining & 34 & 0 \\
\hline Intermediate staining & 13 & 9 \\
\hline Strong staining & 0 & 38 \\
\hline IHC score, mean $\pm \mathrm{SE}$ & $3.7 \pm 1.7$ & $9.9 \pm 2.0$ \\
\hline P-value & $<0.05$ & \\
\hline \multicolumn{3}{|l|}{ LDHA expression } \\
\hline No staining & 0 & 0 \\
\hline Weak staining & 25 & 0 \\
\hline Intermediate staining & 22 & 14 \\
\hline Strong staining & 0 & 33 \\
\hline $\mathrm{IHC}$ score, mean $\pm \mathrm{SE}$ & $4.4 \pm 2.5$ & $9.9 \pm 2.2$ \\
\hline P-value & $<0.05$ & \\
\hline \multicolumn{3}{|l|}{ IL-8 expression } \\
\hline No staining & 0 & 0 \\
\hline Weak staining & 44 & 0 \\
\hline Intermediate staining & 3 & 10 \\
\hline Strong staining & 0 & 37 \\
\hline IHC score, mean $\pm \mathrm{SE}$ & $3.1 \pm 1.2$ & $9.4 \pm 1.6$ \\
\hline P-value & $<0.05$ & \\
\hline
\end{tabular}

significantly higher and intracellular ROS level was lower in LoVo-P cells, indicating the function of PRL-3 in improving colorectal cancer cell glycolysis. Moreover, we also found the expression of glycolysis related molecules and enzymes
Glut1, HK2, PKM2 and LDHA were increased in LoVo-P cells, these findings provide evidence that PRL-3 promotes colorectal cancer cell glycolysis.

Our previous research showed the association between PRL-3 and inflammation in tumor microenvironment. Tumor associated macrophages secreted IL-6 and IL-8 enhanced colorectal cancer cells invasion, however, the mechanism remained unclear. Previous research on the association between inflammation and cancer mainly focused on tumor growth, angiogenesis, EMT, invasion, colonization and recruitment (26-31). Besides, activation of several signal pathways was found to be involved in chronic inflammation such as $N F-\kappa B(32)$. Notably, recent studies revealed that activation of $N F-\kappa B$ increased glycolysis in the inflammatory environment (33). In the present study, we integrated the association between IL- 8 and glycolysis. Inflammatory cytokine antibody array showed upregulation of IL-8 in LoVo-P cells, suggesting correlation between PRL-3 and IL-8. Furthermore, glycolysis of colorectal cancer cells was inhibited when IL- 8 was neutralized, and inhibition was more significant in LoVo-P cells, indicating the important role of IL-8 in improving colorectal cancer cell glycolysis by PRL-3. Tumor metabolism reprogram available cell proliferation and even invasion since glycolysis produces more biological materials than oxidative phosphorylation (34). This study confirmed the function of glycolysis in colorectal cancer cell proliferation and invasion. Various research has explored the correlation between glycolysis and metastasis. For example, decreased $\mathrm{pH}$ may facilitate the invasion of tumor cells by promoting adjacent non-tumor cell apoptosis (35), and it has been found that by TGF- $\beta$ dependent regulation of MMP2, lactate promotes tumor migration (36). Moreover, Glut1 was found to be correlated with MMP-2, which is important in degrading the basement membrane and improving cancer cell invasion (37); Hexokinase 2 was found to be a potent factor which is associated with cancer cell migration (17); PKM2 was found to promote cancer cell migration via activation of STAT signal pathway (18). In the present study, when lactate was neutralized or glycolysis related molecules and enzymes were inhibited, colorectal cancer cell invasion was repressed significantly, which is consistent with other research. Furthermore, we showed high expression of PRL-3, Glut1, HK2, PKM2, LDHA and IL-8 in tumor legion of colorectal cancer, and the positive correlation between PRL-3 and other molecules, indicating the possible clinical therapeutic strategies for colorectal cancer patients.

In summary, this study demonstrated that PRL-3 improved glycolysis of colorectal cancer cells via the secretion of IL-8. However, the detailed mechanism is still unknown and will be investigated in our following research.

\section{Acknowledgements}

The present study was supported by the National Natural Science Foundation of Guangdong Province (no. 2016A030313353), the National Natural Science Foundation of China (no. 81602539), the International Science and Technology Cooperation Program of Guangdong Province (no. 2013B051000025) and the Science and Technology Project of Guangdong Province (no. 2015A050502021). 


\section{References}

1. Ferlay J1, Soerjomataram I, Dikshit R, Eser S, Mathers C, Rebelo M, Parkin DM, Forman D and Bray F: Cancer incidence and mortality worldwide: Sources, methods and major patterns in globocan 2012. Int J Cancer 136: E359-E386, 2015.

2. Saha S, Bardelli A, Buckhaults P, Velculescu VE, Rago C, St Croix B, Romans KE, Choti MA, Lengauer C, Kinzler KW et al: A phosphatase associated with metastasis of colorectal cancer. Science 294: 1343-1346, 2001.

3. Al-Aidaroos AQ and Zeng Q: PRL-3 phosphatase and cancer metastasis. J Cell Biochem 111: 1087-1098, 2010.

4. Jiang Y, Liu XQ, Rajput A, Geng L, Ongchin M, Zeng Q, Taylor GS and Wang J: Phosphatase PRL-3 is a direct regulatory target of TGFbeta in colon cancer metastasis. Cancer Res 71: 234-244, 2011.

5. Molleví DG, Aytes A, Padullés L, Martínez-Iniesta M, Baixeras N, Salazar R, Ramos E, Figueras J, Capella G and Villanueva A: PRL-3 is essentially overexpressed in primary colorectal tumours and associates with tumour aggressiveness Br J Cancer 99: 1718-1725, 2008.

6. Lai W, Chen S, Wu H, Guan Y, Liu L, Zeng Y, Zhao H, Jiang J and Chu Z: PRL-3 promotes the proliferation of LoVo cells via the upregulation of KCNN4 channels. Oncol Rep 26: 909-917, 2011.

7. Lai W, Liu L, Zeng Y, Wu H, Xu H, Chen S and Chu Z: KCNN4 channels participate in the EMT induced by PRL-3 in colorectal cancer. Med Oncol 30: 566, 2013.

8. Xu H, Lai W, Zhang Y, Liu L, Luo X, Zeng Y, Wu H, Lan Q and Chu Z: Tumor-associated macrophage-derived IL-6 and IL- 8 enhance invasive activity of lovo cells induced by Prl- 3 in a kcnn4 channel-dependent manner. BMC Cancer 14: 330, 2014.

9. Warburg O, Wind F and Negelein E: The metabolism of tumors in the body. J Gen Physiol 8: 519-530, 1927.

10. Brahimi-Horn MC, Chiche J and Pouysségur J: Hypoxia signalling controls metabolic demand. Curr Opin Cell Biol 19: 223-229, 2007.

11. Martins SF, Amorim R, Viana-Pereira M, Pinheiro C, Costa RF Silva P, Couto C, Alves S, Fernandes S, Vilaça S, et al: Significance of glycolytic metabolism-related protein expression in colorectal cancer, lymph node and hepatic metastasis. BMC Cancer 16: 535, 2016.

12. Chen KY, Liu X, Bu P, Lin CS, Rakhilin N, Locasale JW and Shen X: A metabolic signature of colon cancer initiating cells. Conf Proc IEEE Eng Med Biol Soc 2014: 4759-4762, 2014.

13. Erreni M, Mantovani A and Allavena P: Tumor-associated macrophages (TAM) and inflammation in colorectal cancer. Cancer Microenviron 4: 141-154, 2011.

14. Xu X, Li J, Sun X, Guo Y, Chu D, Wei L, Li X, Yang G, Liu X, Yao L, et al: Tumor suppressor NDRG2 inhibits glycolysis and glutaminolysis in colorectal cancer cells by repressing c-Myc expression. Oncotarget 6: 26161-26176, 2015.

15. Estrella V, Chen T, Lloyd M, Wojtkowiak J, Cornnell HH, Ibrahim-Hashim A, Bailey K, Balagurunathan Y, Rothberg JM, Sloane BF, et al: Acidity generated by the tumor microenvironment drives local invasion. Cancer Res 73: 1524-1535, 2013.

16. Wellberg EA, Johnson S, Finlay-Schultz J, Lewis AS, Terrell KL, Sartorius CA, Abel ED, Muller WJ and Anderson SM: The glucose transporter GLUT1 is required for ErbB2-induced mammary tumorigenesis. Breast Cancer Res 18: 131, 2016.

17. Katagiri M, Karasawa H, Takagi K, Nakayama S, Yabuuchi S, Fujishima F, Naitoh T, Watanabe M, Suzuki T, Unno M, et al: Hexokinase 2 in colorectal cancer: A potent prognostic factor associated with glycolysis, proliferation and migration. Histol Histopathol 1: 11799, 2016.

18. Yang P, Li Z, Fu R, Wu H and Li Z: Pyruvate kinase M2 facilitates colon cancer cell migration via the modulation of STAT3 signalling. Cell Signal 26: 1853-1862, 2014.
19. Xian ZY, Liu JM, Chen QK, Chen HZ, Ye CJ, Xue J, Yang HQ, Li JL, Liu XF and Kuang SJ: Inhibition of LDHA suppresses tumor progression in prostate cancer. Tumour Biol 36: 8093-8100, 2015.

20. Hanahan D and Weinberg RA: Hallmarks of cancer: The next generation. Cell 144: 646-674, 2011.

21. Min SH, Kim DM, Heo YS, Kim HM, Kim IC and Yoo OJ: Downregulation of p53 by phosphatase of regenerating liver 3 is mediated by MDM2 and PIRH2. Life Sci 86: 66-72, 2010.

22. Basak S, Jacobs SBR, Krieg AJ, Pathak N, Zeng Q, Kaldis P, Giaccia AJ and Attardi LD: The metastasis-associated gene Prl-3 is a p53 target involved in cell-cycle regulation. Mol Cell 30: 303-314, 2008

23. Peng L, Xing X, Li W, Qu L, Meng L, Lian S, Jiang B, Wu J and Shou C: PRL-3 promotes the motility, invasion, and metastasis of LoVo colon cancer cells through PRL-3-integrin beta1-ERK1/2 and-MMP2 signaling. Mol Cancer 8: 110, 2009.

24. Yang $\mathbf{J}$ and Weinberg RA: Epithelial-mesenchymal transition: At the crossroads of development and tumor metastasis. Dev Cell 14: 818-829, 2008.

25. Ming J, Liu N, Gu Y, Qiu X and Wang EH: PRL-3 facilitates angiogenesis and metastasis by increasing ERK phosphorylation and up-regulating the levels and activities of Rho-A/C in lung cancer. Pathology 41: 118-126, 2009.

26. Grivennikov SI and Karin M: Dangerous liaisons: STAT3 and NF-kappaB collaboration and crosstalk in cancer. Cytokine Growth Factor Rev 21: 11-19, 2010.

27. Zumsteg A and Christofori G: Corrupt policemen: Inflammatory cells promote tumor angiogenesis. Curr Opin Oncol 21: 60-70, 2009.

28. Wu Y, Deng J, Rychahou PG, Qiu S, Evers BM and Zhou BP: Stabilization of snail by NF-kappaB is required for inflammationinduced cell migration and invasion. Cancer Cell 15: 416-428, 2009.

29. Wu Y and Zhou BP: Inflammation: A driving force speeds cancer metastasis. Cell Cycle 8: 3267-3273, 2009.

30. Nguyen DX, Bos PD and Massagué J: Metastasis: From dissemination to organ-specific colonization. Nat Rev Cancer 9: 274-284, 2009.

31. Luo JL, Maeda S, Hsu LC, Yagita H and Karin M: Inhibition of NF-kappaB in cancer cells converts inflammation- induced tumor growth mediated by TNFalpha to TRAIL-mediated tumor regression. Cancer Cell 6: 297-305, 2004.

32. Greten FR, Eckmann L, Greten TF, Park JM, Li ZW, Egan LJ, Kagnoff MF and Karin M: IKKbeta links inflammation and tumorigenesis in a mouse model of colitis-associated cancer. Cell 118: 285-296, 2004.

33. Dáňová K, Klapetková A, Kayserová J, Šedivá A, Špíšek R and Jelínková LP: NF-кB, p38 MAPK, ERK1/2, mTOR, STAT3 and increased glycolysis regulate stability of paricalcitol/dexamethasone-generated tolerogenic dendritic cells in the inflammatory environment. Oncotarget 16: 14123-14138, 2015.

34. Vander Heiden MG, Cantley LC and Thompson CB: Understanding the Warburg effect: The metabolic requirements of cell proliferation. Science 324: 1029-1033, 2009.

35. Williams AC,Collard TJ and Paraskeva C: An acidic environment leads to p53 dependent induction of apoptosis in human adenoma and carcinoma cell lines: Implications for clonal selection during colorectal carcinogenesis. Oncogene 18: 3199-3204, 1999.

36. Baumann F, Leukel P, Doerfelt A, Beier CP, Dettmer K, Oefner PJ, Kastenberger M, Kreutz M, Nickl-Jockschat T, Bogdahn U, et al: Lactate promotes glioma migration by TGF-beta2-dependent regulation of matrix metalloproteinase-2. Neuro Oncol 11: 368-380, 2009.

37. Ito S, Fukusato T, Nemoto T, Sekihara H, Seyama $\mathrm{Y}$ and Kubota S: Coexpression of glucose transporter 1 and matrix metalloproteinase-2 in human cancers. J Natl Cancer Inst 94: 1080-1091, 2002. 